\title{
Low-energy collisions between electrons and $\mathrm{BeH}^{+}$: cross sections and rate coefficients for all the vibrational states of the ion
}

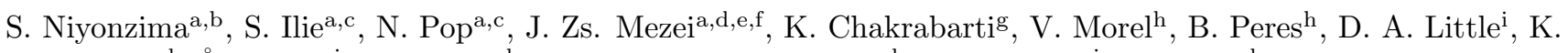 \\ Hassouni $^{\mathrm{d}}$, A.. Larson ${ }^{\mathrm{j}}$, A. E. Orel ${ }^{\mathrm{k}}$, D. Benredjem ${ }^{\mathrm{e}}$, A. Bultel ${ }^{\mathrm{h}}$, J. Tennyson ${ }^{\mathrm{i}}$, D. Reiter ${ }^{\mathrm{l}}$, I. F. Schneider ${ }^{\mathrm{a}, \mathrm{e}, *}$ \\ ${ }^{a}$ Laboratoire Ondes et Milieux Complexes CNRS-Université du Havre- Normandie Université, 76058 Le Havre, France \\ ${ }^{b}$ Département de Physique, Faculté des Sciences, Université du Burundi, B.P. 2700 Bujumbura, Burundi \\ ${ }^{c}$ Fundamental of Physics for Engineers Department, Politehnica University Timisoara, 300223 Timisoara, Romania \\ ${ }^{d}$ Laboratoire des Sciences des Procédés et des Matériaux, CNRS-Université Paris 13-USPC, 93430 Villetaneuse, France \\ ${ }^{e}$ Laboratoire Aimé-Cotton, CNRS-Université Paris-Sud-ENS Cachan-Université Paris-Saclay, 91405 Orsay, France \\ ${ }^{f}$ Institute of Nuclear Research, Hungarian Academy of Sciences, Debrecen, Hungary \\ ${ }^{g}$ Department of Mathematics, Scottish Church College, Calcutta 700 006, India \\ ${ }^{h}$ CORIA CNRS-Université de Rouen-Université Normandie, F-76801 Saint-Etienne du Rouvray, France \\ ${ }^{i}$ Department of Physics and Astronomy, University College London, WC1E 6BT London, UK \\ ${ }^{j}$ Department of Physics, Stockholm University, AlbaNova University Center, S-106 91 Stockholm, Sweden \\ ${ }^{k}$ Department of Chemical Engineering and Materials Science, University of California, Davis, California 95616, USA \\ ${ }^{l}$ Institute of Energy and Climate Research-Plasma Physics, Forschungszentrum Jülich GmbH Association EURATOM-FZJ, Partner in \\ Trilateral Cluster, 52425 Jülich, Germany
}

\begin{abstract}
We provide cross sections and Maxwell rate coefficients for reactive collisions of slow electrons with $\mathrm{BeH}^{+}$ions on all the eighteen vibrational levels $\left(X^{1} \Sigma^{+}, v_{i}^{+}=0,1,2, \ldots, 17\right)$ using a Multichannel Quantum Defect Theory (MQDT) - type approach. These data on dissociative recombination, vibrational excitation and vibrational de-excitation are relevant for magnetic confinement fusion edge plasma modeling and spectroscopy, in devices with beryllium based main chamber materials, such as the International Thermonuclear Experimental Reactor (ITER) and the Joint European Torus (JET). Our results are presented in graphical form and as fitted analytical functions, the parameters of which are organized in tables.
\end{abstract}

\footnotetext{
* Corresponding author.

Email address: ioan.schneider@univ-lehavre.fr (I. F. Schneider)
} 


\section{Contents}

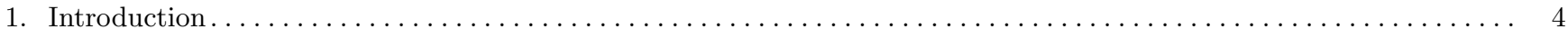

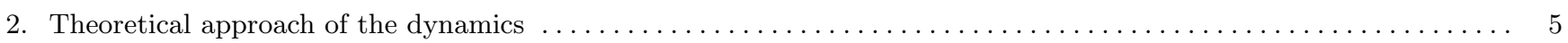

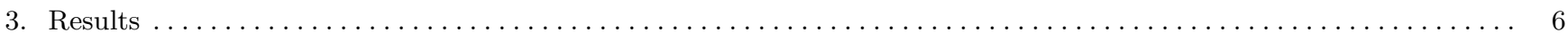

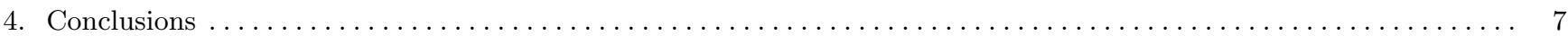

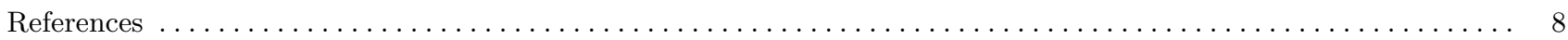

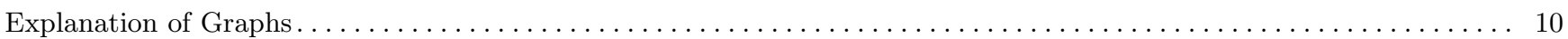

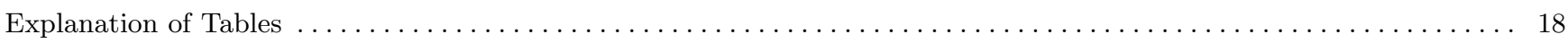

Tables

1. List of the parameters used in Eq.(10) for the DR Maxwell rate coefficients of $\mathrm{BeH}^{+}\left(v_{i}^{+}=0,1,2,3, \ldots, 17\right)$

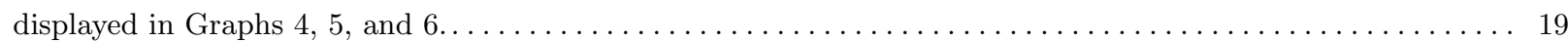

2. List of the parameters used in Eq.(10) for the VE and VdE Maxwell rate coefficients of $\mathrm{BeH}^{+}\left(v_{i}^{+}=0\right.$ and 1$)$

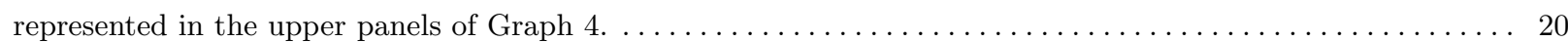

3. List of the parameters used in Eq.(10) for the VE and VdE Maxwell rate coefficients of $\mathrm{BeH}^{+}\left(v_{i}^{+}=2\right.$ and 3$)$

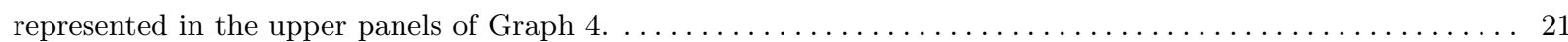

4. List of the parameters used in Eq.(10) for the VE and VdE Maxwell rate coefficients of $\mathrm{BeH}^{+}\left(v_{i}^{+}=4\right.$ and 5)

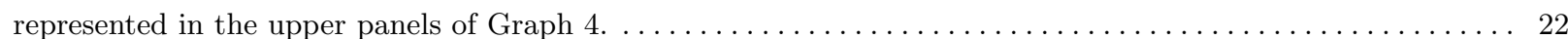

5. List of the parameters used in Eq.(10) for the VE and VdE Maxwell rate coefficients of $\mathrm{BeH}^{+}\left(v_{i}^{+}=6\right.$ and 7$)$

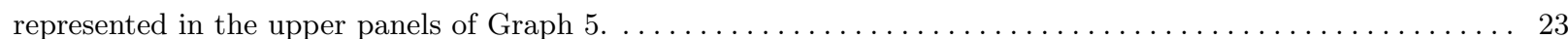

6. List of the parameters used in Eq.(10) for the VE and VdE Maxwell rate coefficients of $\mathrm{BeH}^{+}\left(v_{i}^{+}=8\right.$ and 9$)$

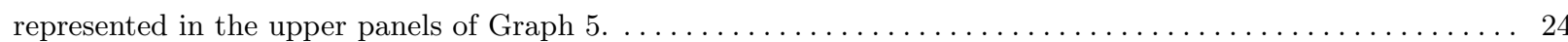

7. List of the parameters used in Eq.(10) for the VE and VdE Maxwell rate coefficients of $\mathrm{BeH}^{+}\left(v_{i}^{+}=10\right.$ and 11$)$

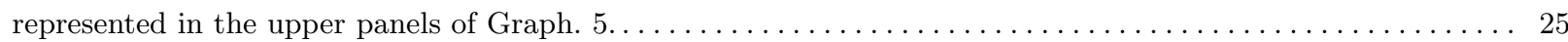

8. List of the parameters used in Eq.(10) for the VE and VdE Maxwell rate coefficients of $\mathrm{BeH}^{+}\left(v_{i}^{+}=12\right.$ and 13$)$

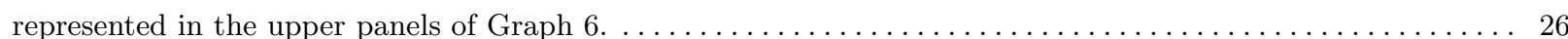

9. List of the parameters used in Eq.(10) for the VE and VdE Maxwell rate coefficients of $\mathrm{BeH}^{+}\left(v_{i}^{+}=14\right.$ and 15)

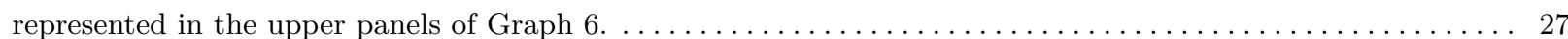

10. List of the parameters used in Eq.(10) for the VE and VdE Maxwell rate coefficients of $\mathrm{BeH}^{+}\left(v_{i}^{+}=16\right.$ and 17)

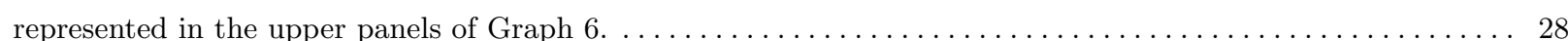

Graphs

1. Dissociative recombination cross sections of ground $\left(v_{i}^{+}=0\right)$ and excited $\left(v_{i}^{+}=1, \ldots, 5\right) \mathrm{BeH}^{+}$in its electronic ground state. Direct mechanism: dashed thick line, total (direct and indirect) mechanism: continuous thin line. 11

2. Dissociative recombination cross sections of excited $\left(v_{i}^{+}=6,7, \ldots, 11\right) \mathrm{BeH}^{+}$in its electronic ground state. Direct mechanism: dashed thick line, total (direct and indirect) mechanism: continuous thin line.

3. Dissociative recombination cross sections of excited $\left(v_{i}^{+}=12,13, \ldots, 17\right) \mathrm{BeH}^{+}$in its electronic ground state. Direct mechanism: dashed thick line, total (direct and indirect) mechanism: continuos thin line.

4. Dissociative recombination (DR, thick line), vibrational excitation (VE, thin lines) and vibrational de-excitation (VdE, symbols and thick lines) Maxwell rate coefficients of ground $\left(v_{i}^{+}=0\right)$ and excited $\left(v_{i}^{+}=1, \ldots, 5\right) \mathrm{BeH}^{+}$ in its electronic ground state (total mechanism). For VE, since the rate coefficients decrease monotonically with the excitation, the lowest final vibrational quantum number of the target is indicated only, and the lower panels extend the range down to $10^{-14} \mathrm{~cm}^{3} / \mathrm{s}$. 
5. Dissociative recombination (DR, thick line), vibrational excitation (VE, thin lines) and vibrational de-excitation (VdE, symbols and thick lines) Maxwell rate coefficients of excited $\left(v_{i}^{+}=7,8, \ldots, 11\right) \mathrm{BeH}^{+}$in its electronic ground state (total mechanism). For VE, since the rate coefficients decrease monotonically with the excitation, the lowest final vibrational quantum number of the target is indicated only, and the lower panels extend the range down to $10^{-14} \mathrm{~cm}^{3} / \mathrm{s}$.

6. Dissociative recombination (DR, thick line), vibrational excitation (VE, thin lines) and vibrational de-excitation (VdE, symbols and thick lines) Maxwell rate coefficients of excited $\left(v_{i}^{+}=12,13, \ldots, 17\right) \mathrm{BeH}^{+}$in its electronic ground state (total mechanism). For VE, since the rate coefficients decrease monotonically with the excitation, the lowest final vibrational quantum number of the target is indicated only, and the lower panels extend the

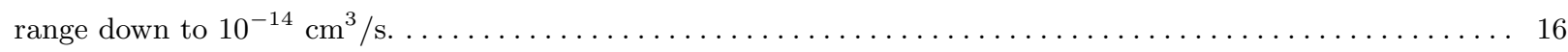

7. Global (sum over all the possible final states) Maxwell rate coefficients for dissociative recombination (DR),

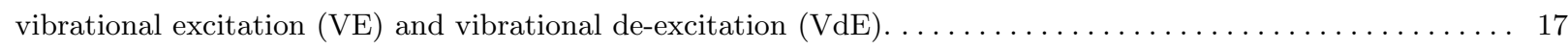




\section{Introduction}

Even though various isotopes of light elements can be coupled to achieve thermonuclear fusion energy release, the next generation of thermonuclear fusion reactors will use deuterium-tritium (D-T) reactions, by far most efficient and accessible plasma fuel for fusion reactors and power plants. As discussed in detail elsewhere [1, 2, 3, 4, 5, 6], beryllium (Be) is meant to enter the composition of the wall of the future fusion devices (ITER). Its performance on preventing tritium retention and, meanwhile, still keeping the benefits of a low Z material (low fuel dilution), is currently being tested in the JET $[3,4]$. According to current plans, tungsten (W) will be the plasma facing material in the high heat flux components (the entire divertor). These materials (Be and W) are expected to provide sufficiently low fuel retention, plasma impurity levels, neutron damage, and sufficient heat removal capabilities in the divertor and then, meet ITER requirements [2]. The key challenge in the use of beryllium as main chamber material for experimental and commercial fusion devices is to understand, predict and control the characteristics of the thermonuclear burning plasma, the plasma edge regimes that result in acceptable erosion performance and the divertor plasma (heat and particle exhaust, impurity control, lifetime).

Due the the low mass ratio between beryllium and the D, T plasma fuel ions, beryllium erodes rather easily under plasma exposure by physical and chemical sputtering, a process which releases $\mathrm{Be}, \mathrm{Be}^{+}$, and other impurities into the plasma. Significant fractions of the eroded beryllium will be transported towards the divertor and will form compounds with the fuel atoms, molecules and/or molecular ions. Therefore, BeH as well as BeD and BeT molecules are expected to appear in a significant (spectroscopically detectable) amount in the edge and divertor plasmas. Various source mechanisms may lead to their formation, either surface or volumetric (particle rearrangement) processes. The particle interchange reaction [7],

$$
\mathrm{Be}^{+}+\mathrm{X}_{2} \longrightarrow \mathrm{BeX}+\mathrm{X}^{+}
$$

where $\mathrm{X}$ denotes one of the fuel atoms $\mathrm{H}$, D or T, was suggested as one, possibly dominant, volumetric BeX formation channel, when $\mathrm{X}_{2}$ is in its vibrational ground state. However, the exo- or endothermicity will greatly depend upon the vibrational state of $\mathrm{X}_{2}$ and other channels, including electron transfer channels, may also be open for BeX formation in fusion divertor plasma conditions. The involved particles - atoms, molecules and molecular ions - follow their often quite complex transport pathways in the edge plasma and take part in the complex reactions determining the plasma composition. Its detailed modeling by taking into account reactions between all present species is necessary, first of all, for interpretation of molecular and atomic line spectroscopy and also to understand and predict the overall plasma edge dynamics and the divertor region behavior in particular.

In principle, the rate of beryllium erosion in fusion devices can be measured by spectroscopical techniques of all the states of the atoms and molecules, so primarily of $\mathrm{Be}, \mathrm{Be}^{+}, \mathrm{Be}_{2}^{+}, \mathrm{BeX}, \mathrm{BeX}^{+}, \mathrm{BeX}_{2}, \mathrm{BeX}_{2}^{+}$. In order to provide a quantitative interpretation of such spectroscopic measurements, one needs a complete set of rate coefficients for excitation, ionization and the various atomic and molecular ions break-up reactions. The inelastic electron-impact processes of vibrationally excited beryllium monohydride $\mathrm{BeH}^{+}$play a key role in the reaction kinetics of low-temperature plasmas in general, and potentially also in certain cold regions of fusion reactor relevant (e.g. the divertor) plasmas. In order to model and diagnose plasmas containing $\mathrm{BeH}^{+}$it is essential to build a complete database of cross-sections and rate coefficients for electron-impact collision processes. Knowing the loss rates of $\mathrm{BeH}^{+}$(and isotopologues) as well as 
absolutely calibrated spectroscopic emission from this molecular ion will allow to draw conclusions also about the $\mathrm{BeH}^{+}$ formation rates.

The $\mathrm{BeH}^{+}$ion is subject to Dissociative Recombination (DR), competed by Vibrational Transitions (VT) - excitation/deexcitation (VE/VdE) - and Dissociative Excitation (DE) respectively [8]:

$$
\mathrm{BeH}^{+}\left(v_{i}^{+}\right)+\mathrm{e}^{-} \longrightarrow \mathrm{Be}+\mathrm{H}, \quad \mathrm{BeH}^{+}\left(v_{f}^{+}\right)+\mathrm{e}^{-}, \quad \mathrm{Be}^{+}+\mathrm{H}+\mathrm{e}^{-},
$$

where $\left(v_{i}^{+}\right) /\left(v_{f}^{+}\right)$are standing for the initial/final vibrational levels of the cation.

Whereas for numerous ions measurements of these reactive collisions have been performed in magnetostatic or electrostatic storage rings (multipass experiments using merged electron and ion beams) [8], this is certainly not the case for $\mathrm{BeH}^{+}$, beryllium being highly toxic.

In the current study, we performed large scale computations of cross sections for the reactive collisions DR, VE and $\mathrm{VdE}$ displayed in eq. (2), as well as of the corresponding rate coefficients.

More specifically, we have used the molecular structure data computed by some of us [9] in order to model the dynamics of these reactions by our stepwise MQDT-method, neglecting the rotational structure and interactions [10, 11, 12, 13]. Whereas our previous works $[9,13]$ restricted to the ground and three lowest vibrationally-excited levels of $\mathrm{BeH}^{+}$, we have extended here our analysis to the whole range of its vibrational states, i.e. up to $v_{i}^{+}=17$.

After briefly reminding the major ideas and steps of our MQDT method, including the main features and parameters of the computational part (section 2), we present the cross sections and rate coefficients below 2.7 eV - dissociation threshold - and below $5000 \mathrm{~K}$, respectively (section 3). The coefficients appearing in the analytical functions used to fit the rate coefficients are organized in tables.

At higher energy of the incident electron, a further competing process, dissociation excitation, will become effective. The collisional data for this range are the subject of ongoing calculations.

\section{Theoretical approach of the dynamics}

In this paper, we use an MQDT-type method to study the electron-impact collision processes:

$$
\mathrm{BeH}^{+}\left(v_{i}^{+}\right)+\mathrm{e}^{-} \longrightarrow \mathrm{BeH}^{*}, \mathrm{BeH}^{* *} \longrightarrow\left\{\begin{array}{l}
\mathrm{Be}+\mathrm{H} \\
\mathrm{BeH}^{+}\left(v_{f}^{+}\right)+\mathrm{e}^{-}
\end{array}\right.
$$

resulting from the quantum interference between the direct mechanism - involving the doubly excited resonant states $\mathrm{BeH}^{* *}$ - and the indirect one - occurring via Rydberg singly-excited predissociating states BeH*.

A detailed description of our theoretical approach was given in [13]. Its main steps are the following:

i) Building of the interaction matrix $\mathcal{V}$ : It is based on the computed $[9,13]$ Rydberg-valence couplings within a quasi-diabatic representation of molecular states of the neutral system. The matrix elements of this matrix correspond to - and are accordingly indexed with - all the possible pairs of channels. More specifically, for a given electronic total angular momentum quantum number $\Lambda$ and a given symmetry (total electronic spin singlet/triplet) of the neutral, our formalism rely on ionization channels - labelled by the vibrational quantum number of the cation $v^{+}$and the orbital quantum number $l$ of the incident/Rydberg electron - and on dissociation channels labelled $d_{j}$. 
ii) Computation of the reaction matrix $\mathcal{K}$ : it is performed by adopting for the Lippman-Schwinger integral equation the second-order perturbative solution $[14,15,16]$, written in operatorial form as:

$$
\mathcal{K}=\mathcal{V}+\mathcal{V} \frac{1}{E-\boldsymbol{H}_{\mathbf{0}}} \mathcal{V}
$$

$\boldsymbol{H}_{\mathbf{0}}$ being the Hamiltonian of the molecular system under study in which the Rydberg-valence interaction is neglected.

iii) Computation of the eigenchannel wavefunctions: It relies on the eigenvectors and eigenvalues of the reaction matrix $\mathcal{K}$, i.e. the columns of the matrix $\boldsymbol{U}$ and the elements of the diagonal matrix $\tan (\boldsymbol{\eta})$ respectively:

$$
\mathcal{K} U=-\frac{1}{\pi} \tan (\eta) U
$$

where the non-vanishing elements of the diagonal matrix $\boldsymbol{\eta}$ are the phaseshifts introduced into the wavefunctions by the short-range interactions.

iv) Frame transformation from the Born-Oppenheimer representation to the close-coupling one: It is performed via the matrices $\mathcal{C}$ and $\mathcal{S}$, built on the basis of the matrices $\boldsymbol{U}$ and $\boldsymbol{\eta}$ and on the quantum defect characterizing the incident/Rydberg electron, $\mu_{l}^{\Lambda}(R)$.

v) Construction of the generalized scattering matrix $\mathcal{X}$, eventually split in blocks associated to open and/or closed (o and/or c respectively) channels:

$$
\mathcal{X}=\frac{\mathcal{C}+i \mathcal{S}}{\mathcal{C}-i \mathcal{S}} \quad \mathcal{X}=\left(\begin{array}{cc}
\boldsymbol{X}_{\text {oo }} & \boldsymbol{X}_{\text {oc }} \\
\boldsymbol{X}_{\text {co }} & \boldsymbol{X}_{\boldsymbol{c c}}
\end{array}\right)
$$

vi) Construction of the physical scattering matrix $\mathcal{S}$, whose elements link mutually the open channels exclusively, given by [17]:

$$
\boldsymbol{S}=\boldsymbol{X}_{\boldsymbol{o o}}-\boldsymbol{X}_{\boldsymbol{o c}} \frac{1}{X_{c c}-\exp (-i 2 \pi \boldsymbol{\nu})} \boldsymbol{X}_{\boldsymbol{c o}}
$$

Here the matrix $\exp (-i 2 \pi \boldsymbol{\nu})$ is diagonal and relies on the effective quantum numbers $\nu_{v^{+}}$associated to the vibrational thresholds of the closed channels.

vii) Computation of the cross-sections: Given the target cation on its level $v_{i}^{+}$, its impact with an electron of energy $\varepsilon$ results either in dissociative recombination or in vibrational excitation/de-excitation according to the formulae:

$$
\begin{gathered}
\sigma_{\operatorname{diss} \leftarrow v_{i}^{+}}=\sum_{\Lambda, s y m} \sigma_{\operatorname{diss} \leftarrow v_{i}^{+}}^{\mathrm{sym}, \Lambda}, \quad \sigma_{\operatorname{diss} \leftarrow v_{i}^{+}}^{\mathrm{sym}, \Lambda}=\frac{\pi}{4 \varepsilon} \rho^{\mathrm{sym}, \Lambda} \sum_{l, j}\left|S_{d_{j}, l v_{i}^{+}}\right|^{2}, \\
\sigma_{v_{f}^{+} \leftarrow v_{i}^{+}}=\sum_{\Lambda, s y m} \sigma_{v_{f}^{+} \leftarrow v_{i}^{+}}^{\mathrm{sym}, \Lambda}, \quad \sigma_{v_{f}^{+} \leftarrow v_{i}^{+}}^{\mathrm{sym}, \Lambda}=\frac{\pi}{4 \varepsilon} \rho^{\mathrm{sym}, \Lambda} \sum_{l, l^{\prime}}\left|S_{l^{\prime} v_{f}^{+}, l v_{i}^{+}}-\delta_{l^{\prime} l} \delta_{v_{i}^{+} v_{f}^{+}}\right|^{2},
\end{gathered}
$$

where, $\rho^{\mathrm{sym}, \Lambda}$ stands for the ratio between the multiplicity of the involved electronic states of BeH and that of the target, $\mathrm{BeH}^{+}$.

\section{Results}

Using the available molecular data - quasi-diabatic potential energy curves and electronic couplings for ${ }^{2} \Pi,{ }^{2} \Sigma^{+}$and ${ }^{2} \Delta$ states displayed in Figure 1 of [13] (for more details see as well [9, 18]) - we have extended our previous calculations 
- initially restricted to the ground and weakly excited vibrational states - to all vibrational levels (up to $v_{i}^{+}=17$ ) of the ground electronic state. The energy of the electron is inferior to $2.7 \mathrm{eV}$, this value corresponding to the dissociation threshold of the ground state ion.

In all the panels of Graphs 1-6, the vertical green lines mark the energy or the temperature below which the calculations are the most accurate. Indeed, above these thresholds, these calculations neglect the role of the dissociative excitation of the ion. Nevertheless, the data displayed continue to be reasonably correct above the thresholds because this process weakly affects the DR and the VT at low energy/temperature.

In order to illustrate the vibrational dependence of the dissociative recombination and to compare the two mechanisms - direct and total - we display in Graphs 1, 2, and 3 the corresponding cross sections. One may notice that the total (direct coherently added to indirect) cross section is characterized by resonant captures into Rydberg states, but that they do not contribute too much in average.

Similar features characterize the cross sections of competitive processes, VE and VdE, but we do not display them here, restricting ourselves to show only their Maxwell rate coefficients.

Indeed, graphs 4, 5, and 6 give the whole ensemble of rate coefficients available for the state-to-state kinetics of $\mathrm{BeH}^{+}$. They illustrate the dominance of the DR in its $v_{i}^{+}=0-9$ levels at low electronic temperature, while the VdE becomes more important than the other processes for initial vibrational states $v_{i}^{+}>9$.

Graph 7 provides the comparison between the DR rate coefficients and the global - i.e. coming from the sum over all the possible final levels - vibrational transitions rate coefficients. One may notice that the excitation process becomes a notable competitor to DR and VdE above $1000 \mathrm{~K}$ only.

In order to allow the versatile implementation of the rate coefficients shown in Graph 4-6 in kinetics modeling codes, we have fitted them with generalized Arrhenius-type formulas:

$$
k_{\left(B e H^{+}, L\right)}^{P}\left(T_{e}\right)=A_{L} T_{e}^{\alpha_{L}} \exp \left[-\sum_{j=1}^{7} \frac{B_{L}(j)}{j T_{e}^{j}}\right]
$$

over the electron temperature range $100 \mathrm{~K} \leq T_{e} \leq 5000 \mathrm{~K}$, 'P' and 'L' standing for Processes (DR or VT) and Levels $\left(v_{i}^{+}\right.$or $\left.v_{i}^{+} \rightarrow v_{f}^{+}\right)$respectively. The coefficients for Dissociative Recombination (when $\mathrm{P}$ corresponds to DR) $A_{v_{i}^{+}}, \alpha_{v_{i}^{+}}$ and $B_{v_{i}^{+}}(j)$ are displayed in Table 1, and those for Vibrational Transitions (when P corresponds to VT, i.e. VE and VdE) $A_{v_{i}^{+} \rightarrow v_{f}^{+}}, \alpha_{v_{i}^{+} \rightarrow v_{f}^{+}}$and $B_{v_{i}^{+} \rightarrow v_{f}^{+}}(j)$ are given in Tables 2-10. The values interpolated using the equation (10) agree with the MQDT-computed ones within a few percent, and are represented in Graphs 4-6.

\section{Conclusions}

The present paper provides a complete state-to-state information of the $\mathrm{BeH}^{+}$reactive collisions with electrons, illustrating quantitatively the competition between the vibrational transitions and dissociative recombination. We display the cross sections or/and the Maxwell rate coefficients for the molecular ion in all of its initial vibrational states and for the entire range of energies of the incident electron below the ion dissociation threshold.

Arrhenius-type formulas are used in order to fit the rate coefficients as function of $T_{e}$, the electronic temperature. These rate coefficients strongly depend on the initial vibrational level of the molecular ion. 
These data are addressed to the fusion community - being relevant to the modeling of the edge of the fusion plasma as well as for divertor conditions - and, more generally, to the modelers of any beryllium-containing-plasmas, produced in laboratory experiments, industrial processing and natural environments. No experimental work concerning the electron $/ \mathrm{BeH}^{+}$collisions can be found in the literature.

Further studies, devoted to higher energy and consequently taking into account the dissociative excitation [19], as well as others, extending to isotopomers of $\mathrm{BeH}^{+}\left(\mathrm{BeD}^{+}, \mathrm{BeT}^{+}\right)$are the object of ongoing work. All these data, as well as the presently displayed ones will be of huge importance for modeling of the plasma/wall interaction [3].

\section{Acknowledgments}

We acknowledge the French LabEx EMC ${ }^{3}$, via the project PicoLIBS (No. ANR-12-BS05-0011-01), the BIOENGINE project (sponsored by the European fund FEDER and the French CPER), the Fdration de Recherche Fusion par Confinement Magntique - ITER and the European COST Program CM1401 (Our Astrochemical History). AEO acknowledges support from the National Science Foundation, Grant No PHY-11-60611. In addition some of this material is based on work done while AEO was serving at the NSF. ÅL acknowledges support from the Swedish Research council, Grant No. 2014-4164. NP and SI acknowledge the Sectoral Operational Programme Human Resources Development (SOP HRD), ID134378 financed from the European Social Fund and by the Romanian Government.

\section{References}

\section{References}

[1] G. Federici, C. H. Skinner, J. N. Brooks, J. P. Coad, C. Grisolia, A. A. Haasz, A. Hassanein, V. Philipps, C. S. Pitcher, J. Roth, W. R. Wampler and D. G. Whyte, Nucl. Fusion 41 (2001) 1967.

[2] J. Paméla, G. F. Matthews, V. Philipps, R. Kamendje and JET-EFDA Contributors, J. Nucl. Mater. 363-365 (2007) 1.

[3] R. Celiberto, R. K. Janev, and D. Reiter, Plasma Phys. Control. Fusion 54 (2012) 035012.

[4] R. A. Anderl, R. A. Causey, J.W. Davis, R. P. Doerner, G. Federici, A.A. Haasz, G. R. Longhurst, W. R. Wampler and K. L. Wilson, J. Nucl. Mater. 273 (1999) 1.

[5] M. Shimada, D. Campbell, V. Mukhovatov, M. Fujiwara, N. Kirneva, K. Lackner, M. Nagami, V. Pustovitov, N. Uckan, J. Wesley, A. Asakura, A. Costley, A. Donn, E. Doyle, A. Fasoli, C. Gormezano, Y. Gribov, O. Gruber, T. Hender, W. Houlberg, S. Ide, Y. Kamada, A. Leonard, B. Lipschultz, A. Loarte, K. Miyamoto, V. Mukhovatov, T. Osborne, A. Polevoi, and A. Sips, Nucl. Fusion 47 (2007) S1.

[6] J. N. Brooks, D. N. Ruzic, and D. B. Hayden, Fusion Eng. Des. 37 (1997) 455.

[7] D. Nishijima, R. P. Doerner, M. J. Baldwin, G. De Temmerman and E. M. Hollmann, Plasma Phys. Control. Fusion 50 ( 2008) 125007.

[8] I. F. Schneider, O. Dulieu, and J. Robert (editors), EPJ Web of Conferences 84 (2015) (Proceedings of the $9^{\text {th }}$ International Conference 'Dissociative Recombination: Theory, Experiment and Applications', Paris, July 7-12 2013). 
[9] J. B. Roos , M. Larsson, Å. Larson, and A. E. Orel, Phys. Rev. A 80 (2009) 012501.

[10] A. Giusti, J. Phys. B 13 (1980) 3867.

[11] I. F. Schneider, I. Rabadán, L. Carata, L. H. Andersen, A. Suzor-Weiner, and J. Tennyson, J. Phys. B 33 (2000) 4849.

[12] B. Vâlcu, I. F. Schneider, M. Raoult, C. Strömholm, M. Larsson, and A. Suzor-Weiner, Eur. Phys. J. D 1 (1998) 71.

[13] S. Niyonzima, F. Lique, K. Chakrabarti, Å. Larson , A. E. Orel, and I. F. Schneider, Phys. Rev. A 87 (2013) 022713.

[14] V. Ngassam, A. Florescu, L. Pichl, I. F. Schneider, O. Motapon, and A. Suzor-Weiner, Eur. Phys. J. D 26 (2003) 165.

[15] A. I. Florescu, V. Ngassam, I. F. Schneider, and A. Suzor-Weiner, J. Phys. B 36 (2003) 1205.

[16] O. Motapon, M. Fifiring, A. Florescu, F. O. Waffeu Tamo, O. Crumeyrolle, G. Varin-Bréant, A. Bultel, P. Vervisch, J. Tennyson, and I. F. Schneider, Plasma Sources Sci. Technol. 15 (2006) 23.

[17] M. J. Seaton, Rep. Prog. Phys. 46 (1983) 167.

[18] C. Strömholm, I. F. Schneider, G. Sundström, L. Carata, H. Danared, S. Datz, O. Dulieu, A. Källberg, M. af Ugglas, X. Urbain, V. Zengin, A. Suzor-Weiner, and M. Larson, Phys. Rev. A 52 (1995) R4320.

[19] K. Chakrabarti, D. R. Backodissa-Kiminou, N. Pop, J. Zs. Mezei, O. Motapon, F. Lique, O. Dulieu, A. Wolf and I. F. Schneider, Phys. Rev. A 87 (2013) 022702. 


\section{Explanation of Graphs}

Graph 1-3. Dissociative recombination cross sections for all the vibrational levels of $\mathrm{BeH}^{+}$in its ground electronic state.
Ordinate
Cross section in $\mathrm{cm}^{2}$
Abscissa
Collision (electron) energy in $\mathrm{eV}$
Black solid line
Total (direct and indirect) process
Red dashed line
Direct process
Green vertical line
Precision limit, for details see the text

Graph 4-6. Dissociative recombination, vibrational excitation and de-excitation Maxwell rate coefficients for all the vibrational levels of $\mathrm{BeH}^{+}$in its ground electronic state.
Ordinate
Maxwell rate coefficient in $\mathrm{cm}^{3} \cdot \mathrm{s}^{-1}$
Abscissa
Electron temperature in $\mathrm{K}$
Thick black line
DR Maxwell rate coefficient
Thin colored lines
Vibrational excitation rate coefficients
Colored lines with symbols
Vibrational de-excitation rate coefficients
Green vertical line
Precision limit, for details see the text

Graph 7. Global (summed-up for all possible final states) dissociative recombination, vibrational excitation and de-excitation Maxwell rate coefficients for $\mathrm{BeH}^{+}$in its ground electronic state.

Ordinate

Abscissa
Maxwell rate coefficient in $\mathrm{cm}^{3} \cdot \mathrm{s}^{-1}$

Electron temperature in $\mathrm{K}$ 


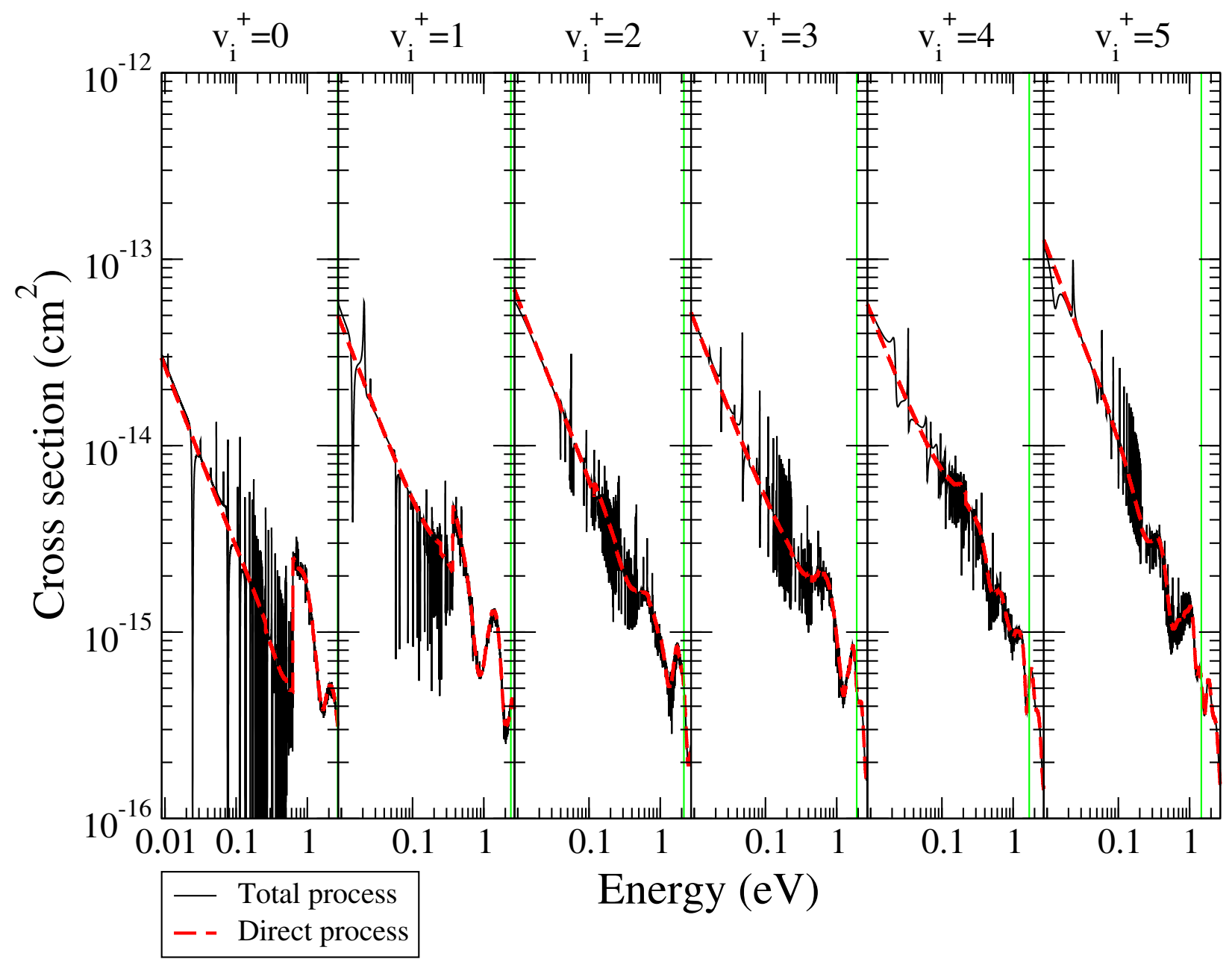

Graph 1. Dissociative recombination cross sections of ground $\left(v_{i}^{+}=0\right)$ and excited $\left(v_{i}^{+}=1, \ldots, 5\right) \mathrm{BeH}^{+}$in its electronic ground state. Direct mechanism: dashed thick line, total (direct and indirect) mechanism: continuous thin line. 


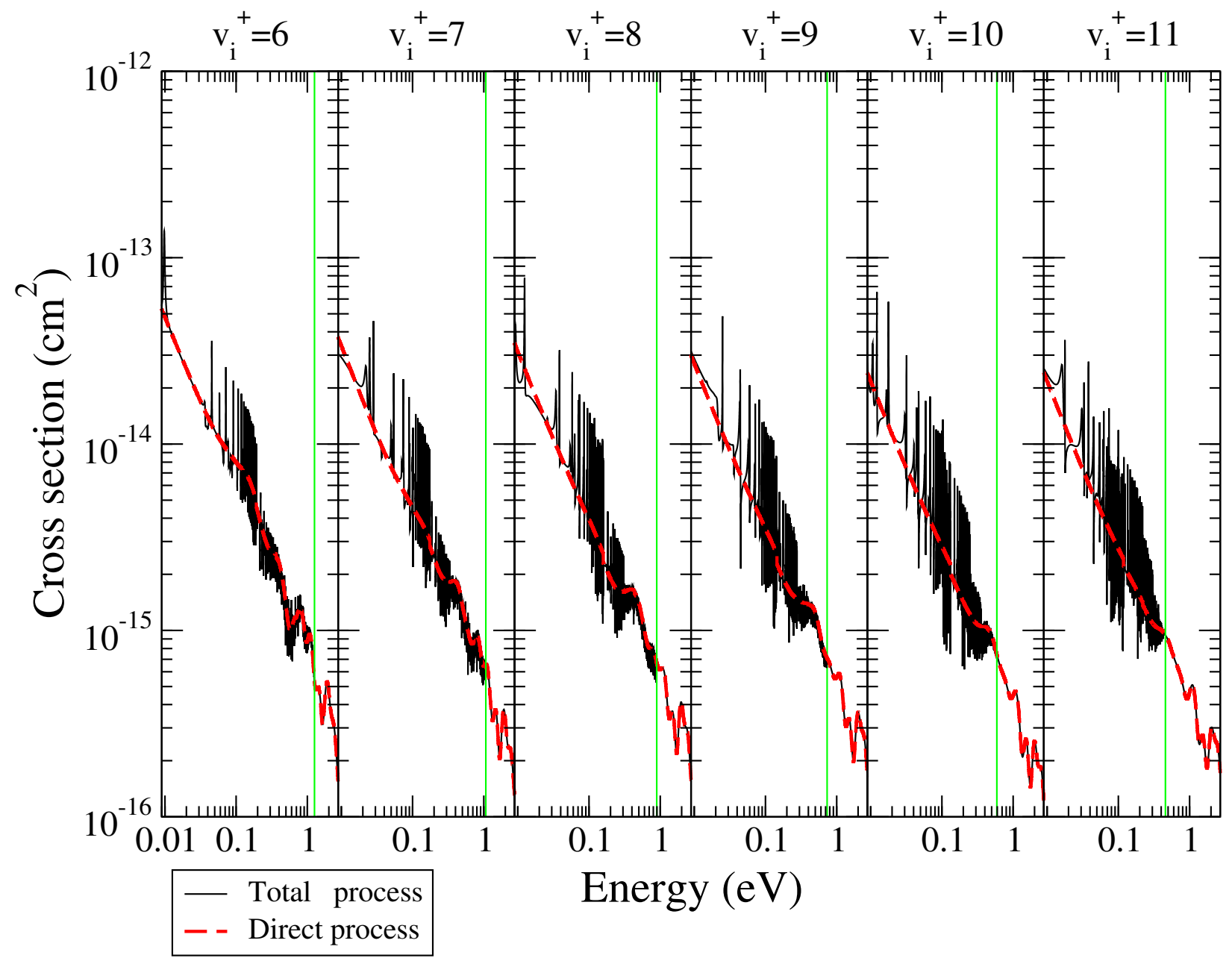

Graph 2. Dissociative recombination cross sections of excited $\left(v_{i}^{+}=6,7, \ldots, 11\right) \mathrm{BeH}^{+}$in its electronic ground state. Direct mechanism: dashed thick line, total (direct and indirect) mechanism: continuous thin line. 


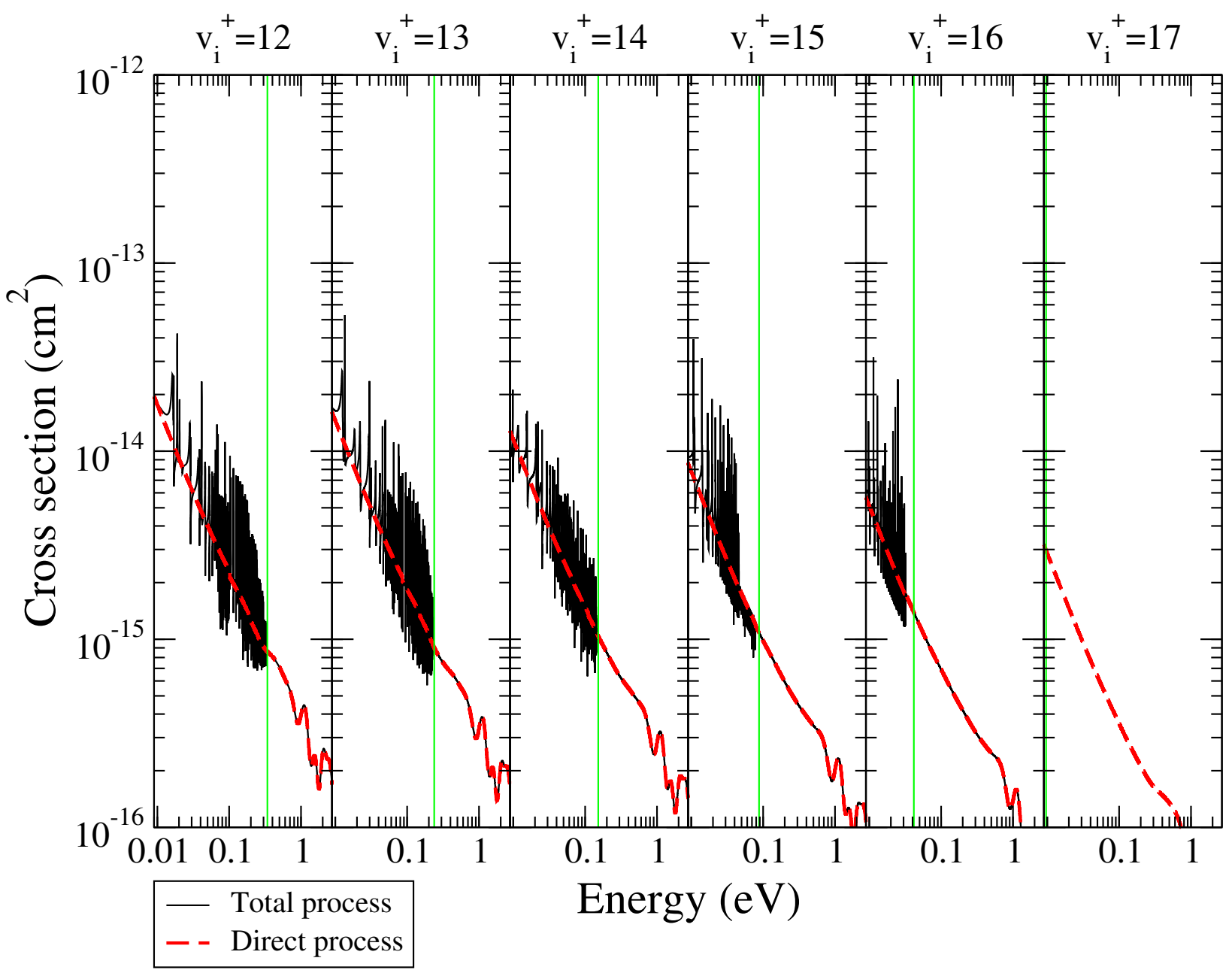

Graph 3. Dissociative recombination cross sections of excited $\left(v_{i}^{+}=12,13, \ldots, 17\right) \mathrm{BeH}^{+}$in its electronic ground state. Direct mechanism: dashed thick line, total (direct and indirect) mechanism: continuos thin line. 


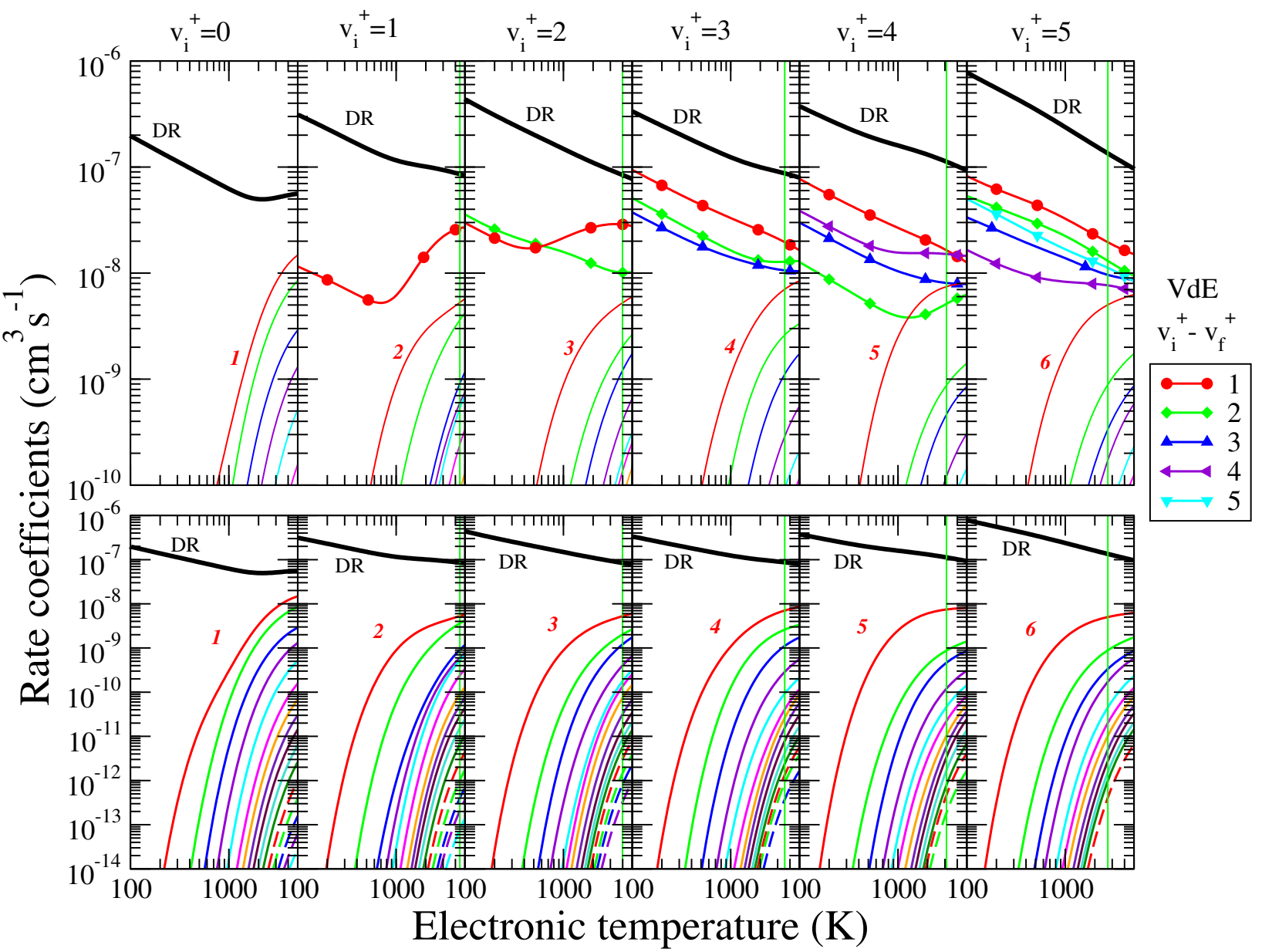

Graph 4. Dissociative recombination (DR, thick line), vibrational excitation (VE, thin lines) and vibrational de-excitation (VdE, symbols and thick lines) Maxwell rate coefficients of ground $\left(v_{i}^{+}=0\right)$ and excited $\left(v_{i}^{+}=1, \ldots, 5\right) \mathrm{BeH}^{+}$in its electronic ground state (total mechanism). For VE, since the rate coefficients decrease monotonically with the excitation, the lowest final vibrational quantum number of the target is indicated only, and the lower panels extend the range down to $10^{-14} \mathrm{~cm}^{3} / \mathrm{s}$. 


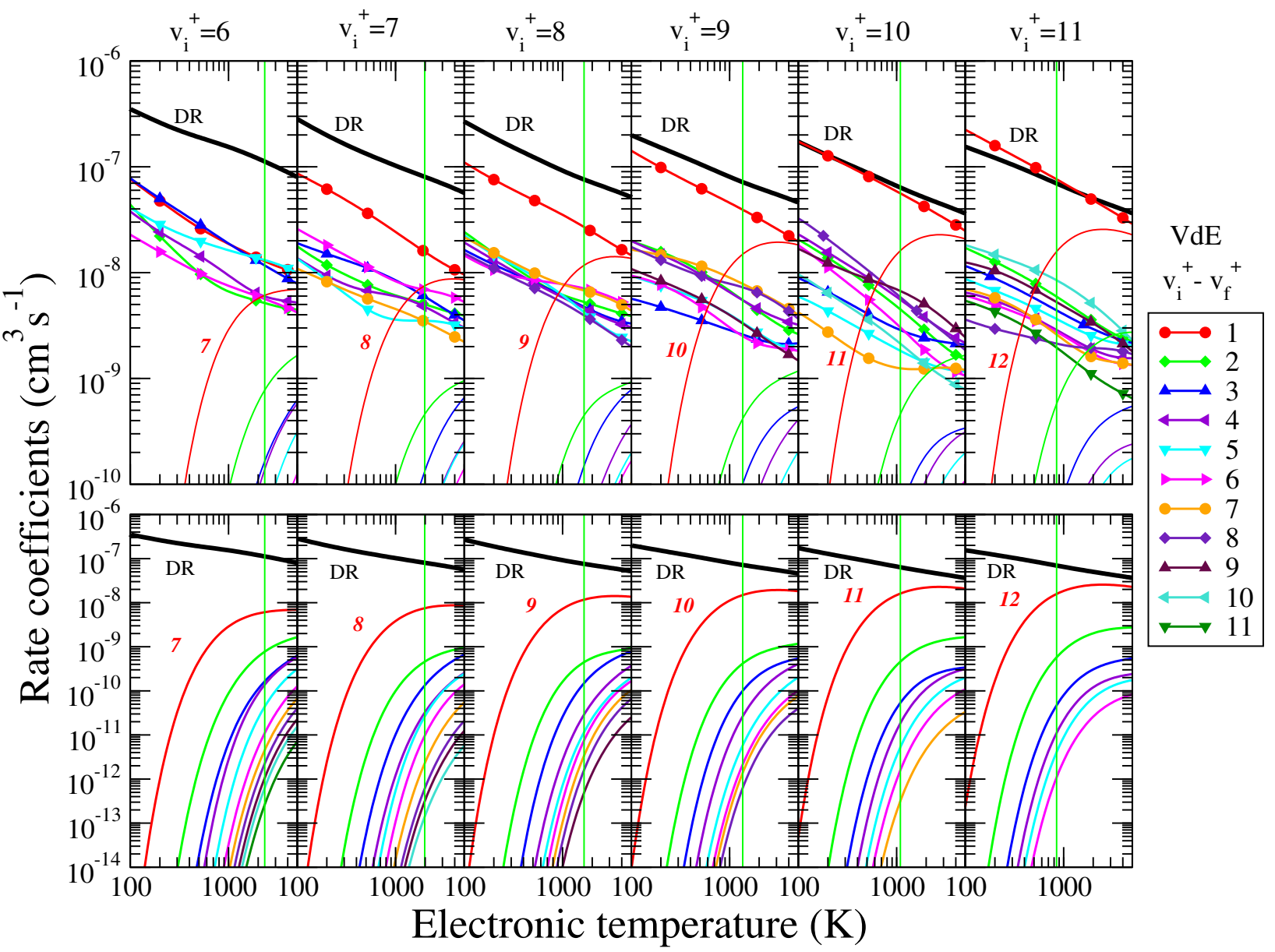

Graph 5. Dissociative recombination (DR, thick line), vibrational excitation (VE, thin lines) and vibrational de-excitation (VdE, symbols and thick lines) Maxwell rate coefficients of excited $\left(v_{i}^{+}=7,8, \ldots, 11\right) \mathrm{BeH}^{+}$in its electronic ground state (total mechanism). For VE, since the rate coefficients decrease monotonically with the excitation, the lowest final vibrational quantum number of the target is indicated only, and the lower panels extend the range down to $10^{-14} \mathrm{~cm}^{3} / \mathrm{s}$. 


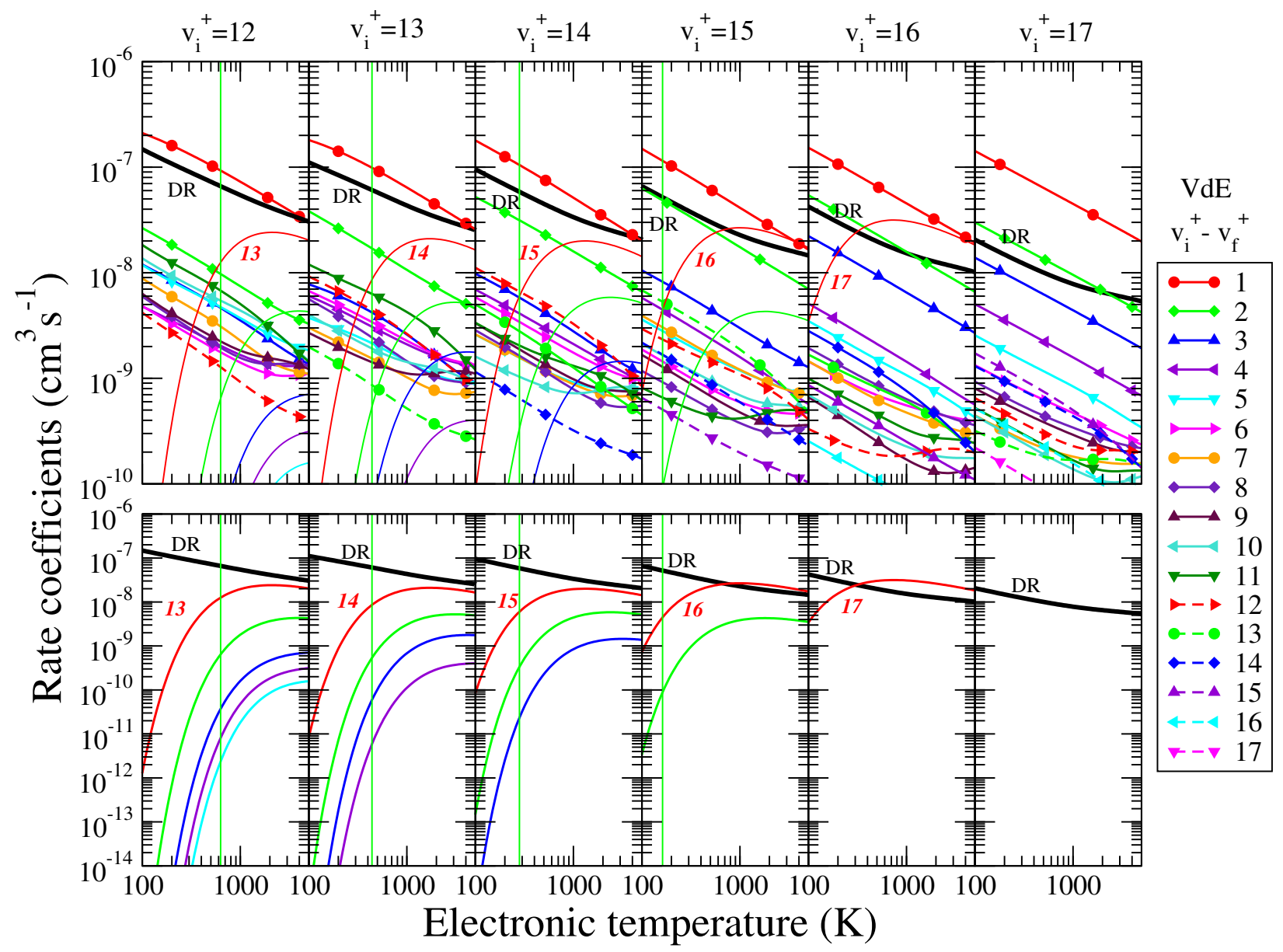

Graph 6. Dissociative recombination (DR, thick line), vibrational excitation (VE, thin lines) and vibrational de-excitation (VdE, symbols and thick lines) Maxwell rate coefficients of excited $\left(v_{i}^{+}=12,13, \ldots, 17\right) \mathrm{BeH}^{+}$in its electronic ground state (total mechanism). For VE, since the rate coefficients decrease monotonically with the excitation, the lowest final vibrational quantum number of the target is indicated only, and the lower panels extend the range down to $10^{-14} \mathrm{~cm}^{3} / \mathrm{s}$. 


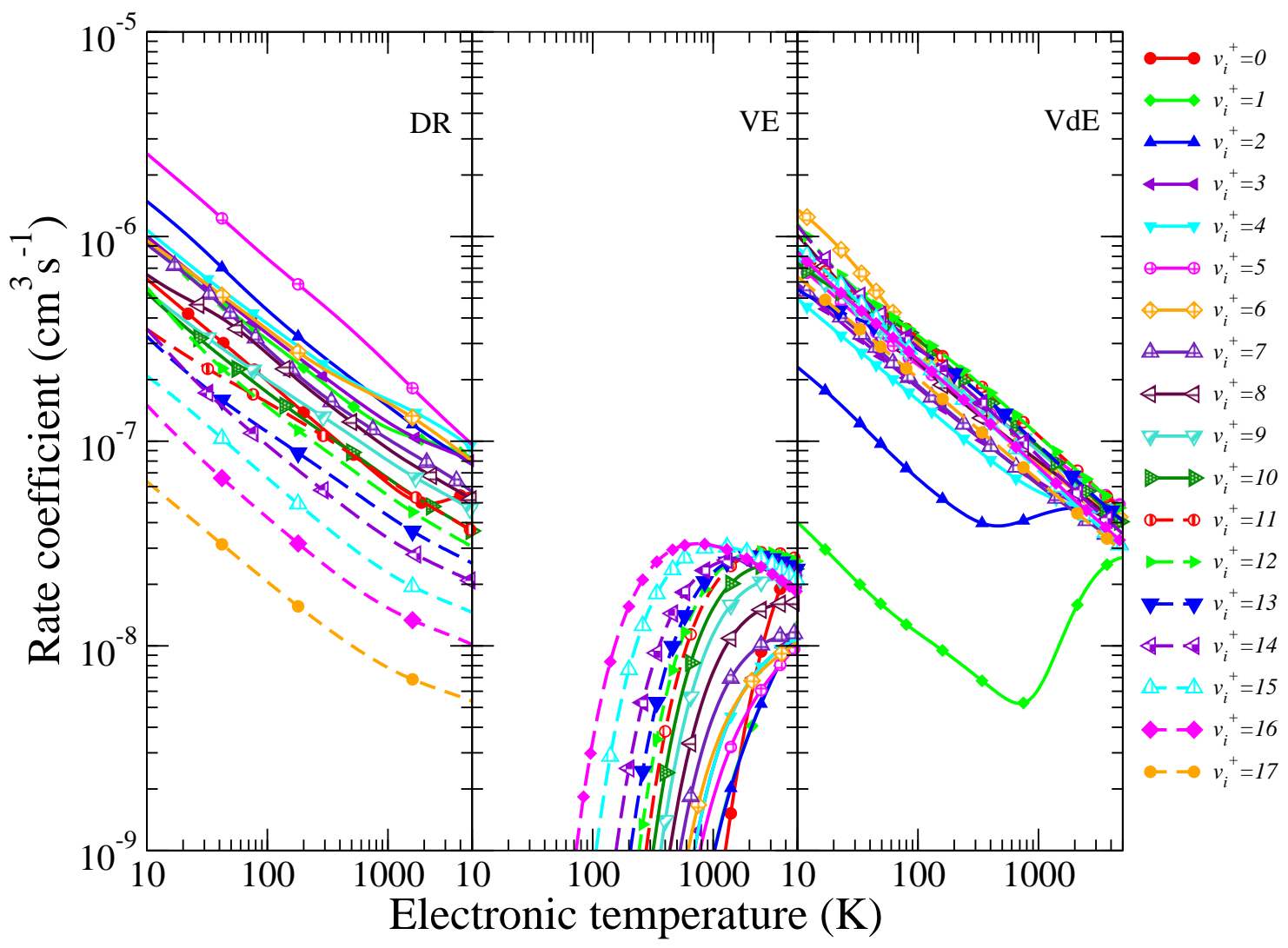

Graph 7. Global (sum over all the possible final states) Maxwell rate coefficients for dissociative recombination (DR), vibrational excitation (VE) and vibrational de-excitation (VdE). 


\section{Explanation of Tables}

Table 1 List of fitting parameters according to eq. (10) with ' $P$ ' $=$ ' $D R$ ' and ' $L '=v_{i}^{+}$' calculated for dissociative recombination for all vibrational levels of the ground electronic state of $B e H^{+}$.
$v_{i}^{+}$
Initial vibrational level of $\mathrm{BeH}^{+}$
$A_{v_{i}^{+}}, \alpha_{v_{i}^{+}}, B_{v_{i}^{+}}(j=1, \ldots, 7)$
Fitting parameters

Tables 2-10 List of fitting parameters according to eq. (10) with ' $P$ ' $=$ ' $V E$ ' or ' $V d E$ ' and ' $L$ ' $=v_{i}^{+} \rightarrow v_{f}^{+}$' calculated for vibrational transitions (excitation and de-excitation) between the vibrational levels of the ground electronic state of $\mathrm{BeH}^{+}$.
$v_{i}^{+} \rightarrow v_{f}^{+}$
Vibrational transition of $\mathrm{BeH}^{+}$
$v_{i}^{+} \rightarrow v_{f}^{+}$
Stands for VdE
$A_{v_{i}^{+} \rightarrow v_{f}^{+}}, \alpha_{v_{i}^{+} \rightarrow v_{f}^{+}}, B_{v_{i}^{+} \rightarrow v_{f}^{+}}(j=1, \ldots, 7)$
Fitting parameters 


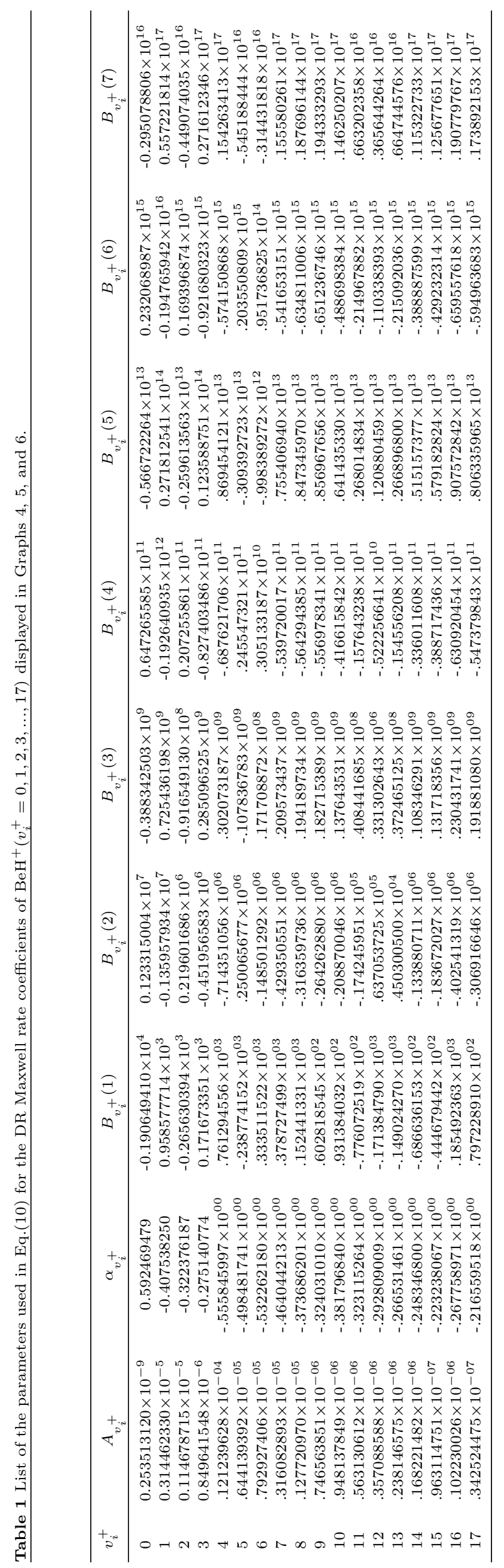




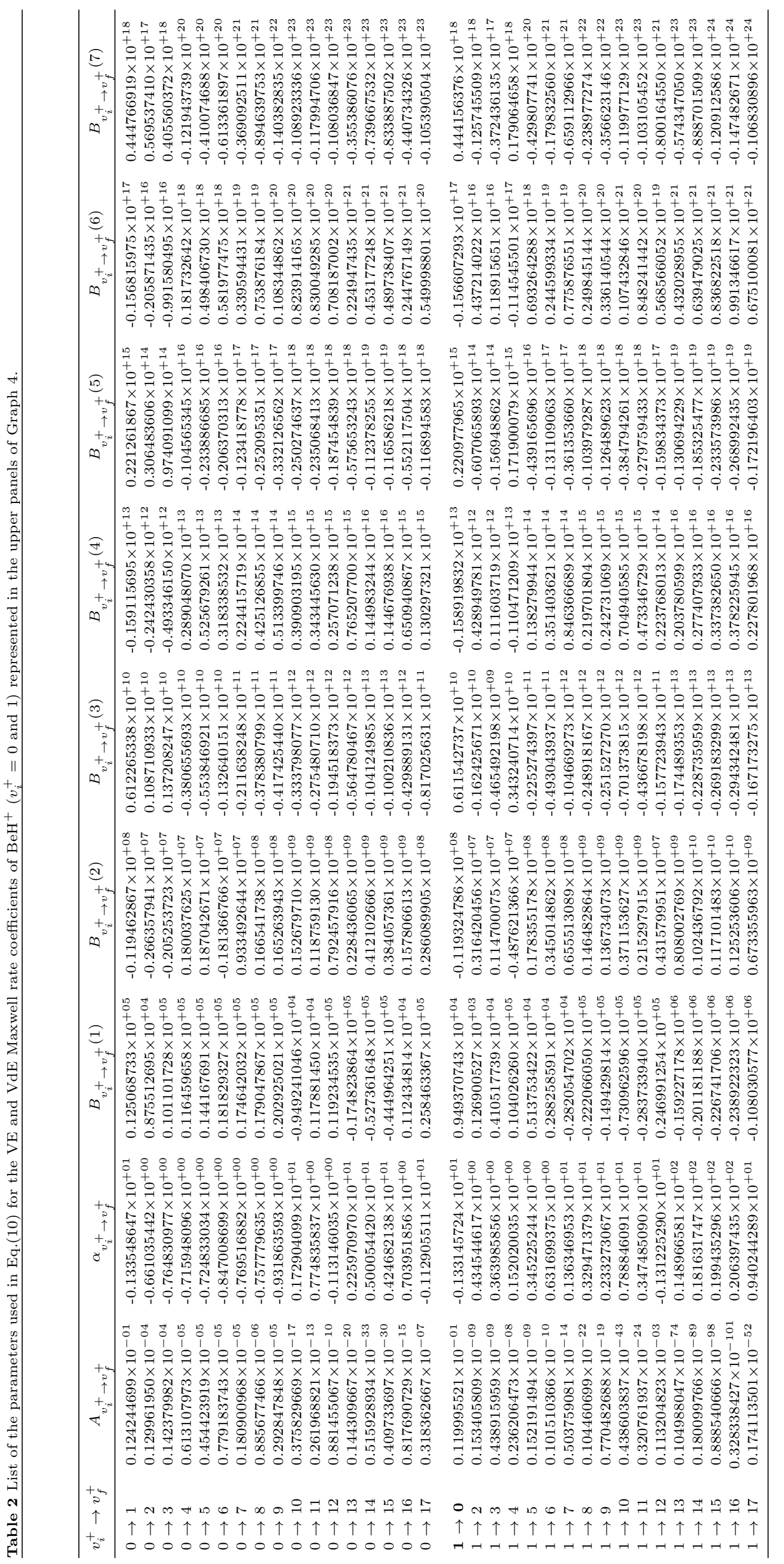




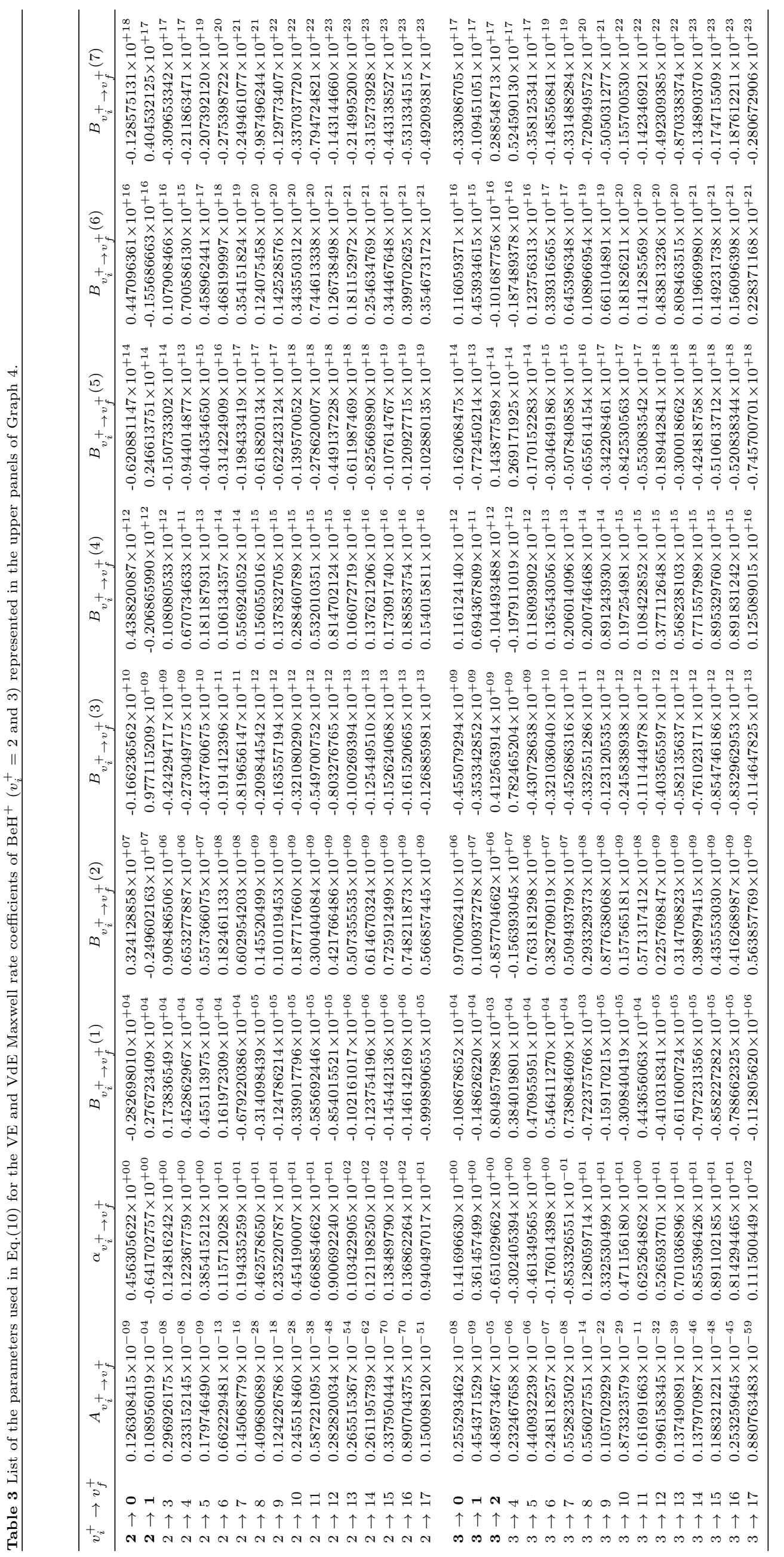




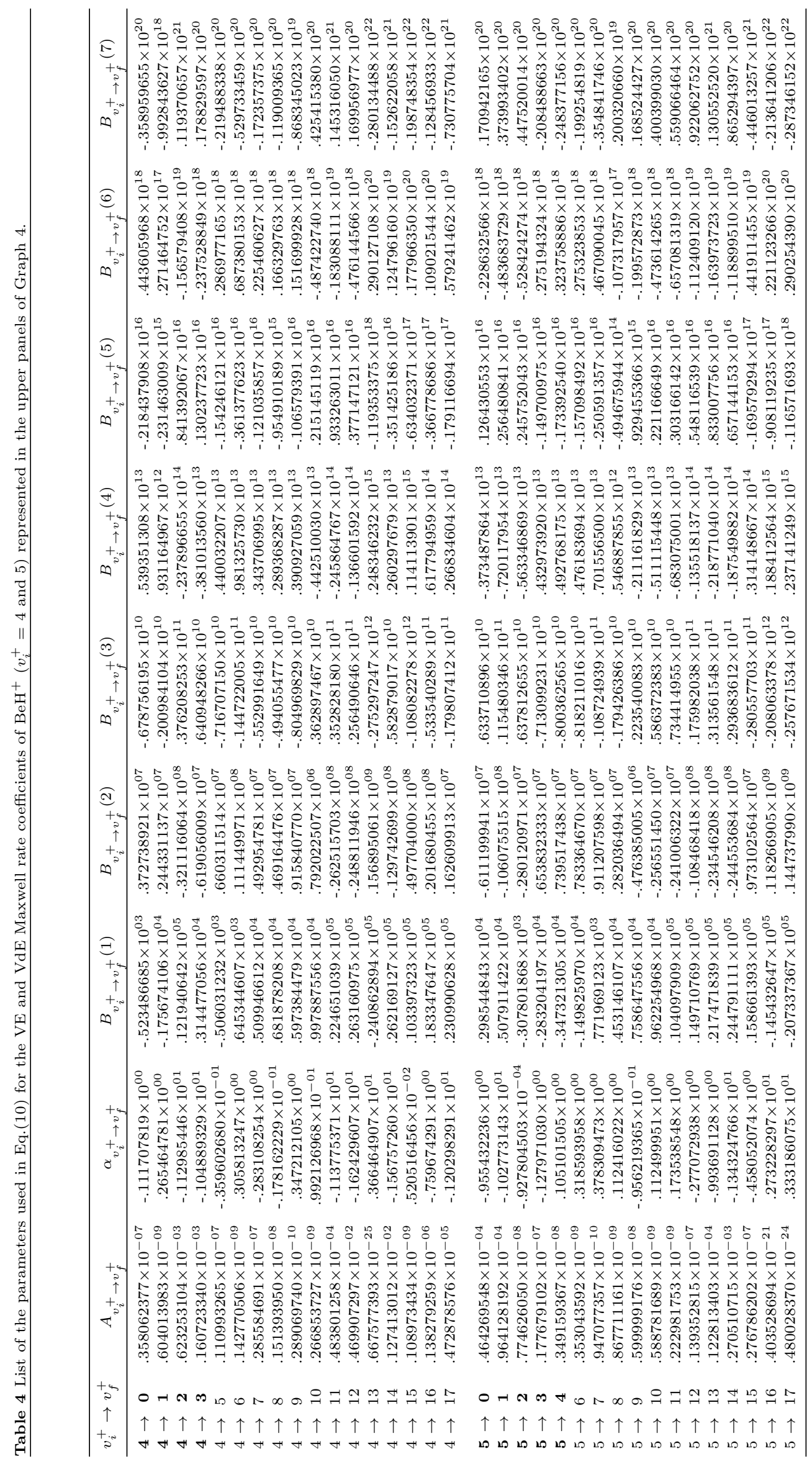




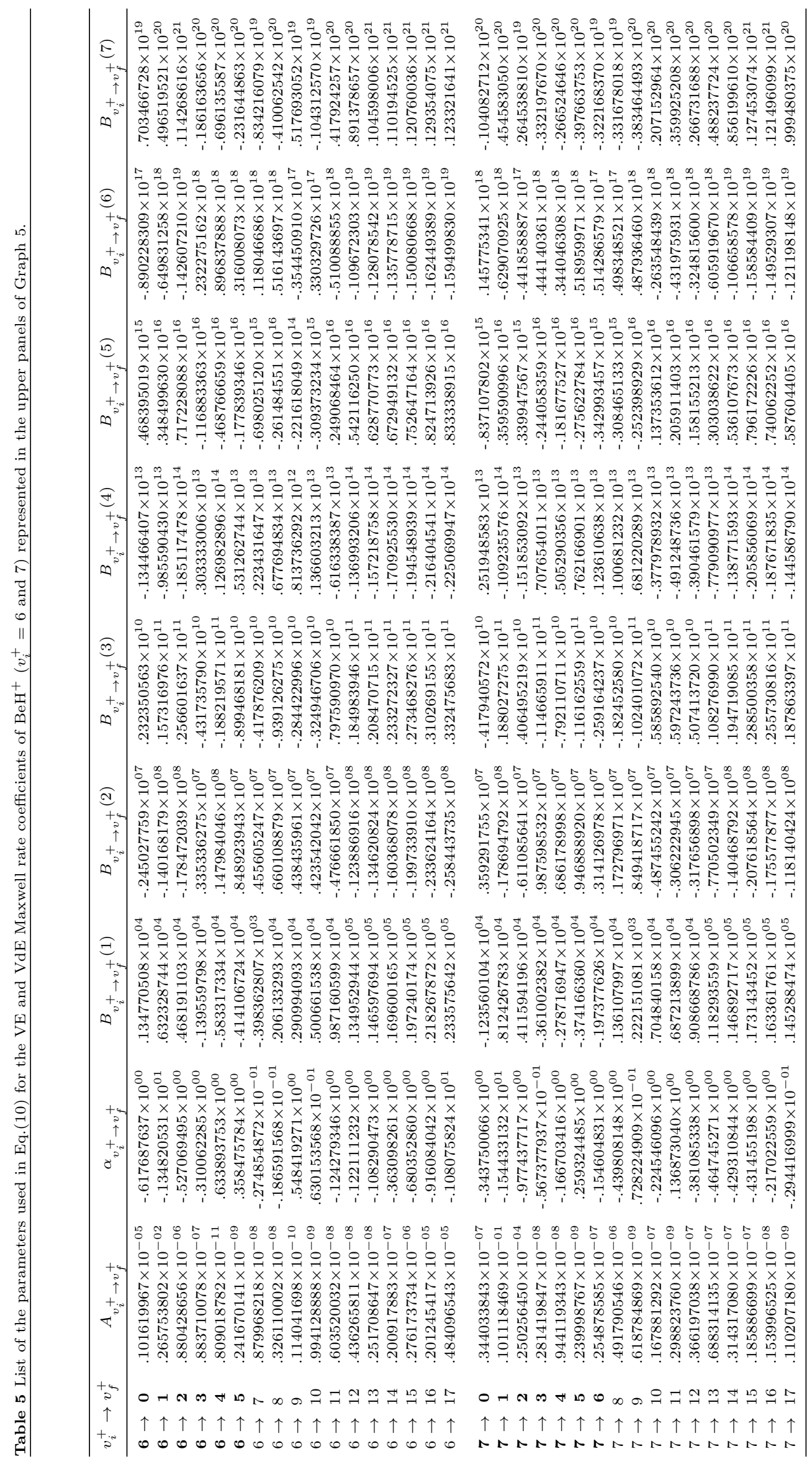




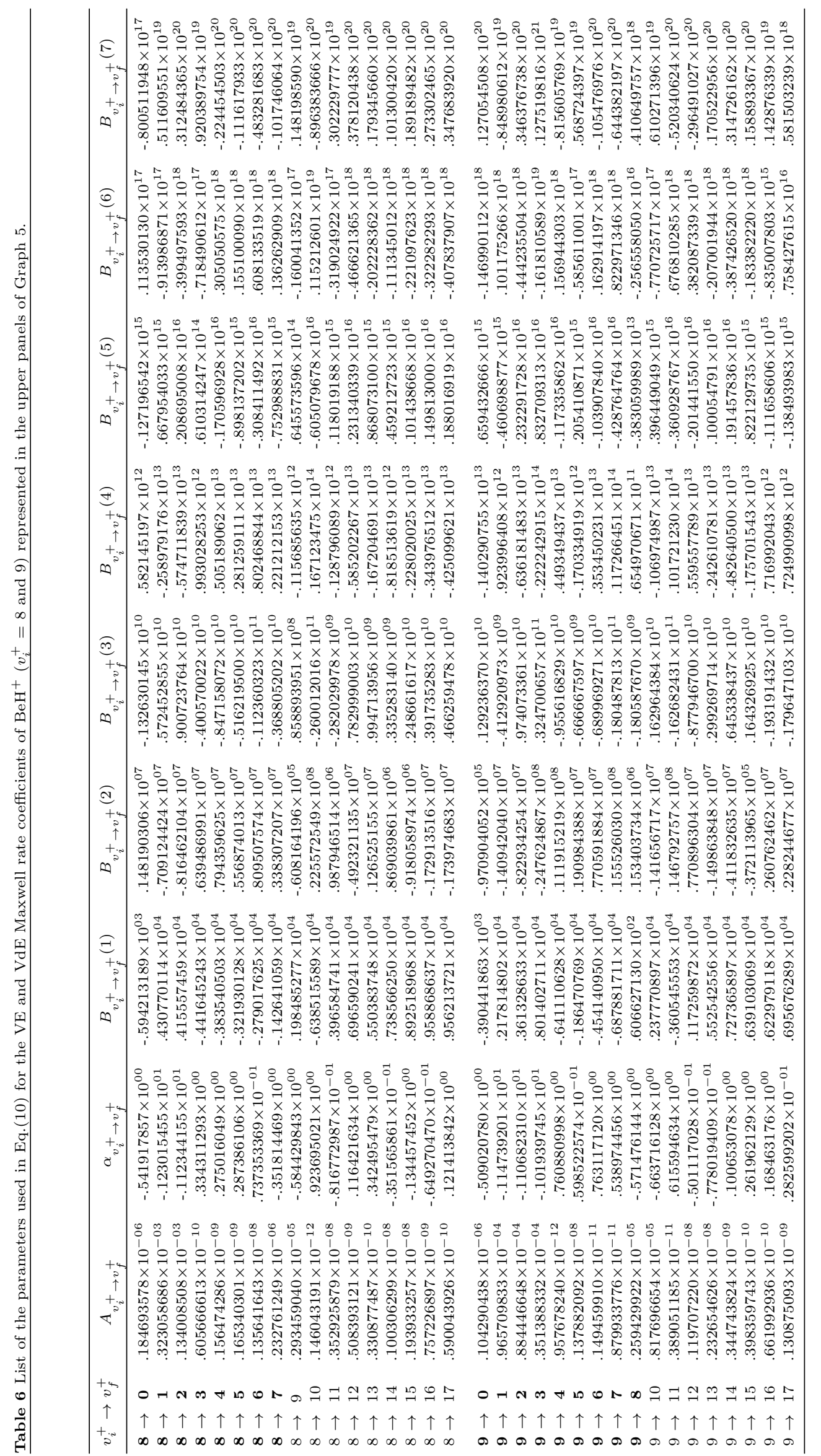




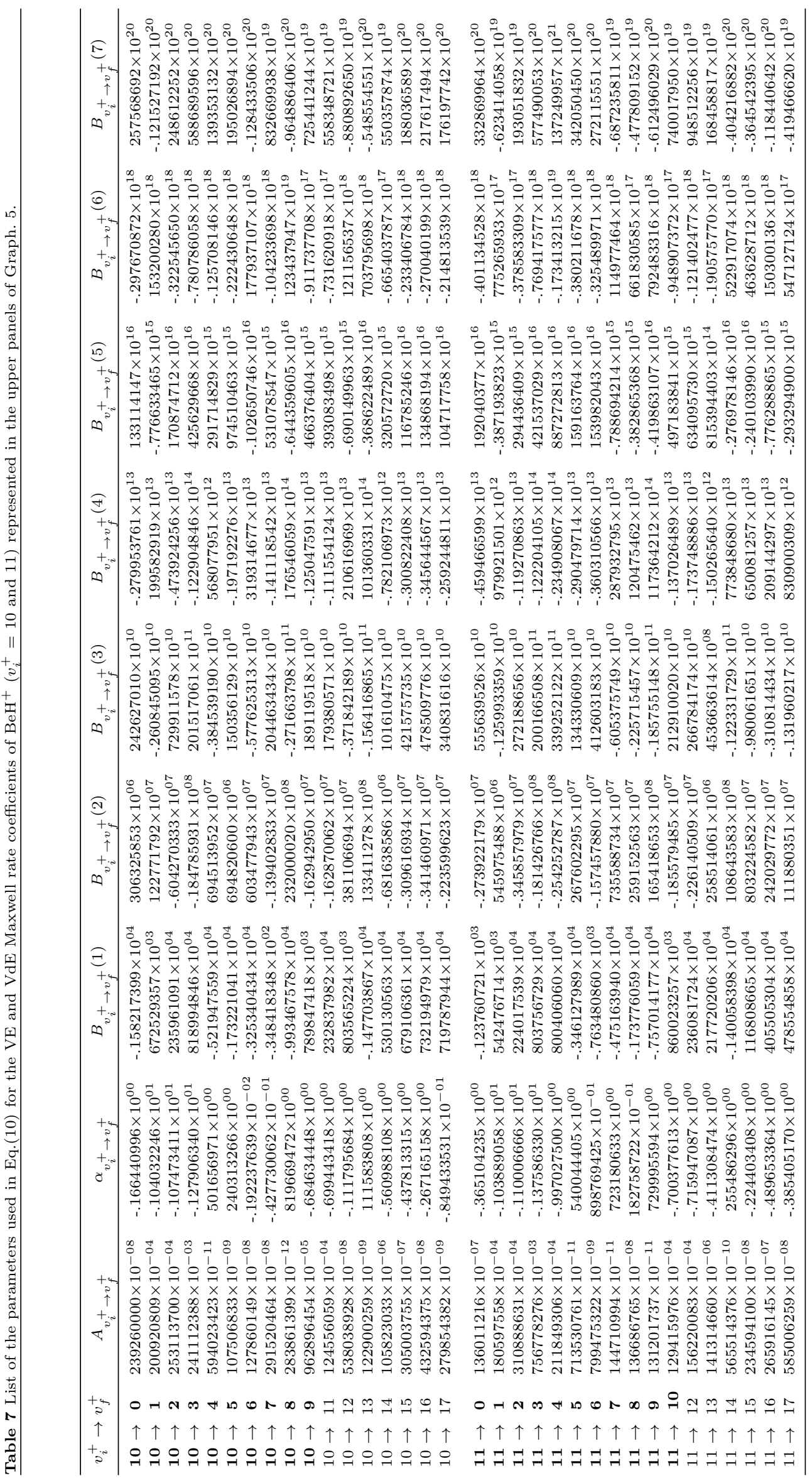




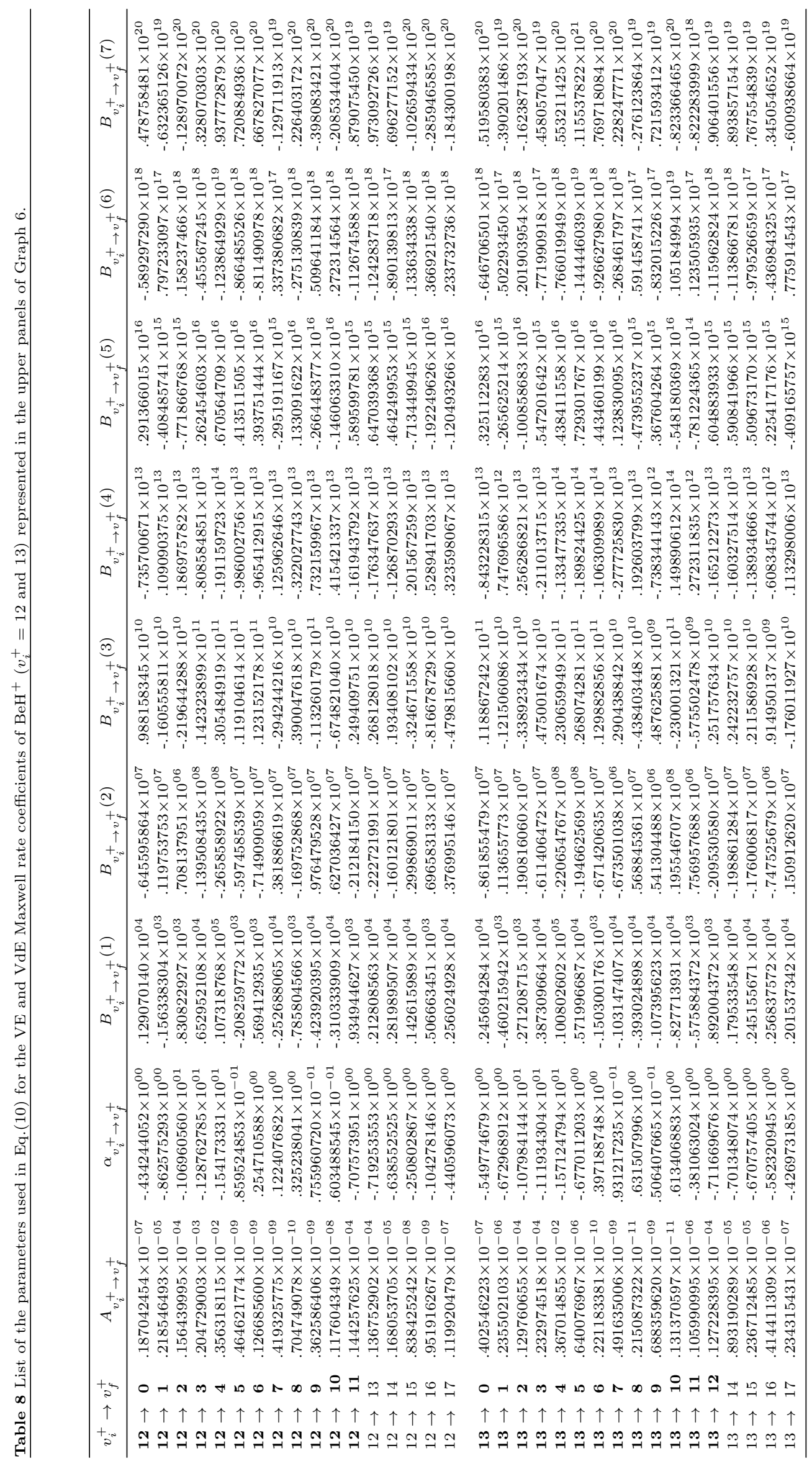




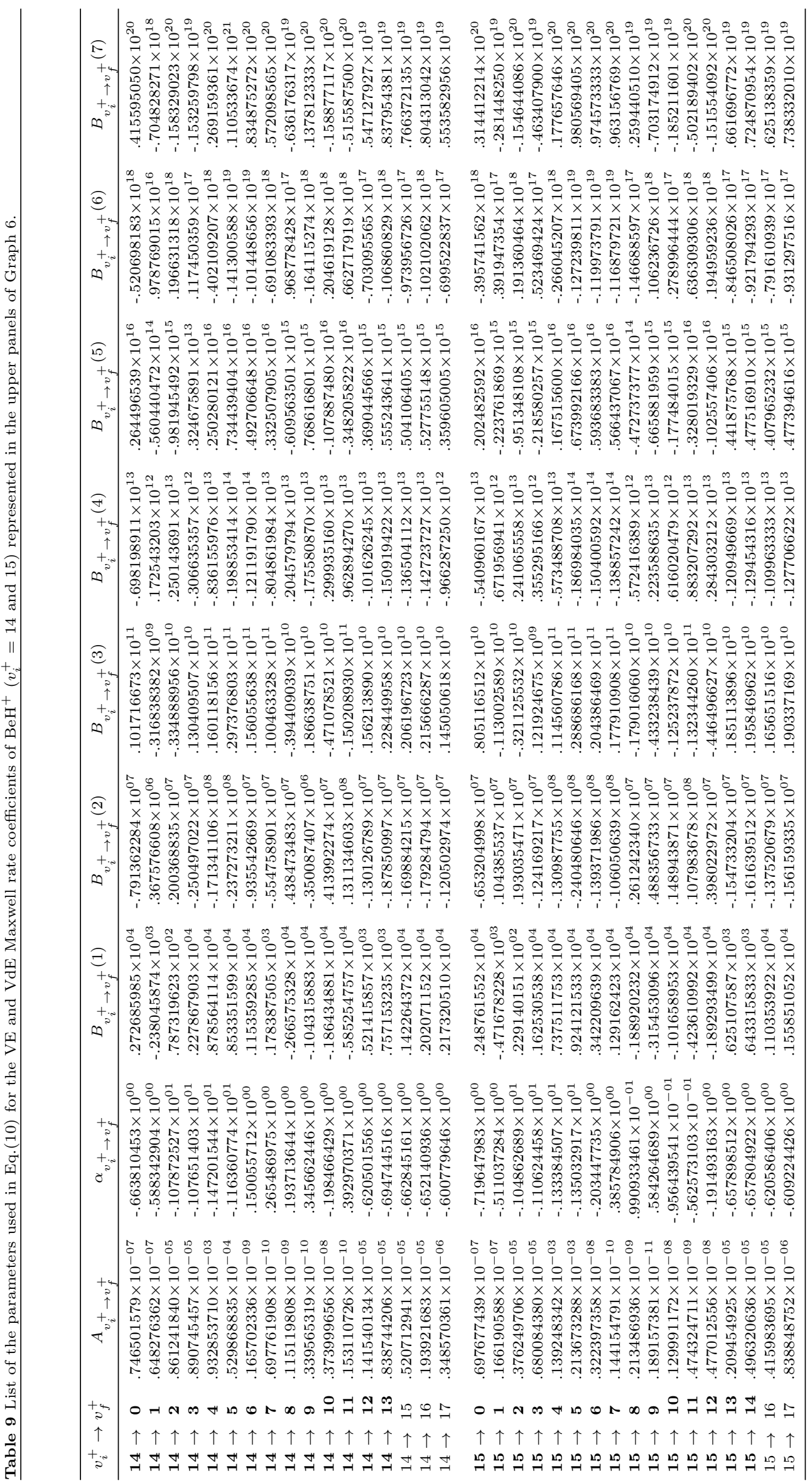




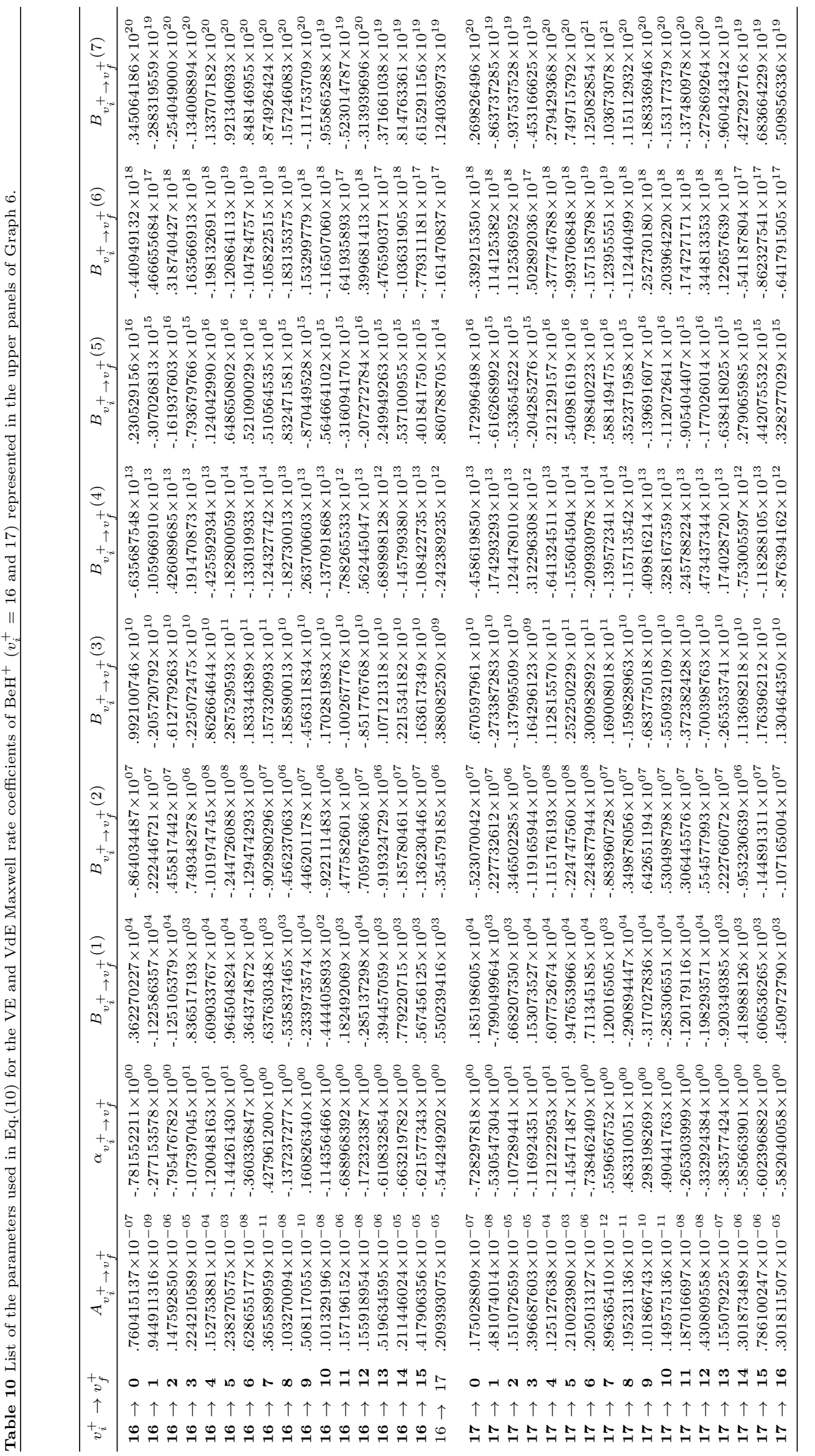

